Proceedings of the 11th International Conference

on Computational and Mathematical Methods

in Science and Engineering, CMMSE 2011

26-30 June 2011.

\title{
Combinatorial structures of three vertices and Lie algebras
}

\author{
J. Cáceres ${ }^{1}$, M. Ceballos ${ }^{2}$, J. Núñez ${ }^{2}$, M.L. Puertas ${ }^{1}$ and Á.F. Tenorio ${ }^{3}$ \\ ${ }^{1}$ Dpto. de Estadística y Matemática Aplicada, Universidad de Almería. \\ ${ }^{2}$ Dpto. de Geometría y Topología, Facultad de Matemáticas. Universidad de Sevilla. \\ ${ }^{3}$ Dpto. de Economía, Métodos Cuantitativos e Historia Económica, Escuela \\ Politécnica Superior. Universidad Pablo de Olavide.
}

emails: jcaceres@ual.es, mceballos@us.es, jnvaldes@us.es, mpuertas@ual.es, aftenorio@upo.es

\begin{abstract}
In this paper, we characterize digraphs of 3 vertices associated with Lie algebras according to isomorphism classes of these associated Lie algebras. At this respect, we introduce and implement two algorithmic methods: the first is devoted to draw the digraph associated with a given Lie algebra and the second allows us to determine if a given digraph is associated or not with a Lie algebra.
\end{abstract}

Key words: Digraph, Lie algebra, Isomorphism class, Algorithm.

MSC 2000: 17B60, 05C25, 05C20, 05C90, 17B20, $17 B 30$.

\section{Introduction}

Finding new links among different fields of Mathematics has always been one of the most interesting challenges in mathematical research, since it allows to use alternative techniques to solve open problems, improve known theories and reveal new ones. This paper is devoted to link Lie Theory and Graph Theory. On one hand, research on Graph Theory is running in a high level, being used as a very useful tool to deal with other knowledge fields. Regarding this, this work continues the line opened in [1], where a mapping between Lie algebras and combinatorial structures was introduced in order to translate properties of Lie algebras into the language of Graph Theory and vice versa. 
On the other hand, applications of Lie Theory are being considered in fields like Engineering, Physics and Applied Mathematics, for instance, being its research very extensive both theoretically and practically. However, several topics are still unsolved and new alternatives are welcome to work them. In this sense, determining which isomorphism classes there exist for nilpotent and solvable Lie algebras is nowadays an important open problem, especially if we take into account that other types of Lie algebras (like semisimple and simple) were completely classified in 1890 .

The main goal of this paper is to make progress in the relation between graphs and Lie algebras, carrying on with previous papers like $[1,2,3]$. The structure is the following: firstly, we determine all isomorphism classes of Lie algebras admitting configurations of 3 vertices described in [1]. In fact, we characterize the different configurations that correspond to the same isomorphism class. Secondly, we introduce and implement two new algorithmic methods based on the relation between graphs and Lie algebras: one to obtain the digraph associated with a given $n$-dimensional Lie algebra and another to determine if a given digraph is associated with a Lie algebra or not.

In our opinion, the procedures introduced here allow us to advance, make easier and improve the characterization of Lie-algebra isomorphism classes by means of the classification of their associated combinatorial structures (graphs, in this case).

\section{Preliminaries of Lie algebras}

Some preliminary concepts of Lie algebras are recalled, bearing in mind that the reader can consult [4] for a general overview. In this paper, we consider $\mathbb{K}=\mathbb{R}$ or $\mathbb{C}$ and $\mathbb{K}^{*}=\mathbb{K} \backslash\{0\}$.

Definition $1 A$ Lie algebra $\mathfrak{g}$ is a vector space with a second bilinear composition law $[\cdot, \cdot]$ called the bracket product, which satisfies two conditions: $[X, X]=0, \forall X \in \mathfrak{g}$ and $[[X, Y], Z]+[[Y, Z], X]+[[Z, X], Y]=0, \forall X, Y, Z \in \mathfrak{g}$. This last condition is called the Jacobi identity and denoted by $J(X, Y, Z)=0$.

Definition 2 The Lie algebra $\mathfrak{g}$ is semisimple if it does not contain any proper abelian ideals. If $\mathfrak{g}$ is non-abelian with no non-trivial ideals, then it is simple.

Definition 3 The commutator central series and the lower central series of a finitedimensional Lie algebra $\mathfrak{g}$ are, respectively,

$$
\begin{gathered}
\mathcal{C}_{1}(\mathfrak{g})=\mathfrak{g}, \mathcal{C}_{2}(\mathfrak{g})=[\mathfrak{g}, \mathfrak{g}], \ldots, \mathcal{C}_{k}(\mathfrak{g})=\left[\mathcal{C}_{k-1}(\mathfrak{g}), \mathcal{C}_{k-1}(\mathfrak{g})\right], \ldots \quad \text { and } \\
\mathcal{C}^{1}(\mathfrak{g})=\mathfrak{g}, \mathcal{C}^{2}(\mathfrak{g})=[\mathfrak{g}, \mathfrak{g}], \ldots, \mathcal{C}^{k}(\mathfrak{g})=\left[\mathcal{C}^{k-1}(\mathfrak{g}), \mathfrak{g}\right], \ldots
\end{gathered}
$$

Hence, $\mathfrak{g}$ is $(m-1)$-step solvable if there exists $m \in \mathbb{N}$ such that $\mathcal{C}_{m}(\mathfrak{g}) \equiv\{0\}$ and $\mathcal{C}_{m-1}(\mathfrak{g}) \neq\{0\}$. Analogously, $\mathfrak{g}$ is $(m-1)$-step nilpotent if there exists $m \in \mathbb{N}$ such that $\mathcal{C}^{m}(\mathfrak{g}) \equiv\{0\}$ and $\mathcal{C}^{m-1}(\mathfrak{g}) \neq\{0\}$ 


\section{Associating combinatorial structures with Lie algebras}

Given an $n$-dimensional Lie algebra $\mathfrak{g}$ with basis $\mathcal{B}=\left\{e_{i}\right\}_{i=1}^{n}$, the law of $\mathfrak{g}$ with respect to $\mathcal{B}$ is given by its structure constants $c_{i, j}^{k}$ as follows: $\left[e_{i}, e_{j}\right]=\sum_{k=1}^{n} c_{i, j}^{k} e_{k}$. The pair $(\mathfrak{g}, \mathcal{B})$ can be associated with a combinatorial structure by the following method, introduced in [1]:

a) For each $e_{i} \in \mathcal{B}$, one vertex labelled as index $i$ is drawn.

b) Given three vertices $i<j<k$, the full triangle can be drawn. The weights $c_{i, j}^{k}$, $c_{j, k}^{i}$, and $c_{i, k}^{j}$ are assigned to the edges $\{i, j\},\{j, k\}$ and $\{i, k\}$, respectively.

b1) If $c_{i, j}^{k}=c_{j, k}^{i}=c_{i, k}^{j}=0$, then the triangle is not drawn.

b2) If a structure constant is zero, its corresponding edge is drawn using a discontinuous line and called ghost edge.

b3) If two triangles of vertices $\{i, j, k\}$ and $\{i, j, l\}$ with $1 \leq i<j<k<l \leq n$ satisfy $c_{i, j}^{k}=c_{i, j}^{l}$, then the edge $\{i, j\}$ is shared.

c) Given two vertices $i<j$, draw a directed edge from $j$ to $i$ if $c_{i, j}^{i} \neq 0$ or from $i$ to $j$ if $c_{i, j}^{j} \neq 0$.

\section{Digraphs of 3-vertices associated with Lie algebras}

There exist only 4 digraphs of 3 vertices associated with 3-dimensional Lie algebras according to Lemma 3.1 in [1] (see Figure 1). We study their isomorphism classes.

a)

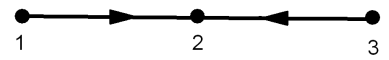

c)

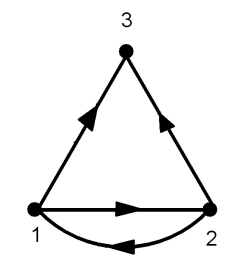

$c^{2}{ }_{1,2} c^{3}{ }_{2,3}+c^{1}{ }_{1,2} c^{3}{ }_{1,3}=0$ b)

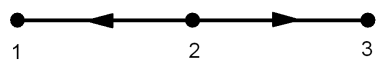

d)

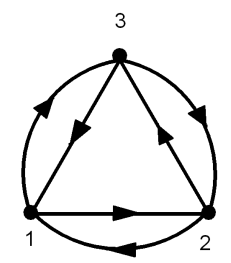

$c_{1,3}^{1} c_{2,3}^{3}+c_{1,2}^{1} c_{2,3}^{2}=0$

$\mathrm{c}^{2}{ }_{1,2} \mathrm{c}_{2,3}^{3}+\mathrm{c}^{1}{ }_{1,2} \mathrm{c}^{3}{ }_{1,3}=0$

$\mathrm{c}^{1}{ }_{1,3} \mathrm{c}_{1,2}^{2}-\mathrm{c}^{3}{ }_{1,3} \mathrm{c}_{2,3}^{2}=0$

Figure 1: Digraphs of 3 vertices associated with Lie algebras. 
Proposition 1 Let us consider the Lie algebra $\mathfrak{g}=\left\langle w_{1}, w_{2}, w_{3}\right\rangle:\left[w_{1}, w_{2}\right]=c_{1,2}^{2} w_{2}$; $\left[w_{2}, w_{3}\right]=c_{2,3}^{2} w_{2}$, with $\left(c_{1,2}^{2}, c_{2,3}^{2}\right) \in \mathbb{K}^{2} \backslash\{(0,0)\}$ associated with the configuration $\left.a\right)$ in Figure 1. Then

i) There exists a basis $\left\{e_{i}\right\}_{i=1}^{3}$ of $\mathfrak{g}$ with respect to which: $\left[e_{1}, e_{2}\right]=e_{2} ;\left[e_{2}, e_{3}\right]=e_{2}$.

ii) There exists a basis $\left\{v_{i}\right\}_{i=1}^{3}$ of $\mathfrak{g}$ with respect to which: $\left[v_{1}, v_{2}\right]=p_{1} v_{1}+p_{2} v_{2}$ with $\left(p_{1}, p_{2}\right) \in \mathbb{K}^{2} \backslash\{(0,0)\}$.

Proof: For i), it suffices to consider the basis change $\phi: \mathfrak{g} \rightarrow \mathfrak{g}$ given by $e_{1}=\phi\left(w_{1}\right)=$ $\frac{1}{c_{1,2}^{2}} w_{1} ; e_{2}=\phi\left(w_{2}\right)=w_{2} ; e_{3}=\phi\left(w_{3}\right)=\frac{1}{c_{2,3}^{2}} w_{3}$.

To prove ii), we consider an arbitrary basis change from an arbitrary basis to the basis given in i) as follows: $e_{i}=\sum_{j=1}^{3} a_{i, j} v_{j}$, with $\left[v_{i}, v_{j}\right]=\sum_{k=1}^{3} d_{i, j}^{k} e_{k}$. Imposing the law given in i) and solving the resulting system, we obtain the law expressed in ii).

Remark 1 Statement ii) in Proposition 1 means that there exists an isomorphism between the two structures of Figure 2, independently of the weights.
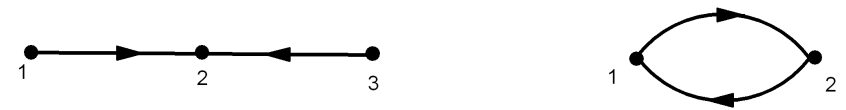

Figure 2: Isomorphism from Proposition 1.

Corollary 1 Lie algebras associated with configuration a) constitutes a unique isomorphism class $\mathfrak{g}_{1}:\left[e_{1}, e_{2}\right]=e_{2},\left[e_{2}, e_{3}\right]=e_{2}$. This class also contains 3-dimensional Lie algebras with center of dimension 1 (associated with a graph having a unique isolated vertex)."

Proposition 2 Let us consider the Lie algebra $\mathfrak{g}=\left\langle w_{1}, w_{2}, w_{3}\right\rangle:\left[w_{1}, w_{2}\right]=c_{1,2}^{1} w_{1}$; $\left[w_{2}, w_{3}\right]=c_{2,3}^{3} w_{3}$, with $c_{1,2}^{1}, c_{2,3}^{3} \in \mathbb{K}^{*}$ associated with the configuration $\left.b\right)$ in Figure 1. Then, there exists a basis $\left\{e_{i}\right\}_{i=1}^{3}$ of $\mathfrak{g}$ verifying $\left[e_{1}, e_{2}\right]=e_{1} ;\left[e_{2}, e_{3}\right]=p e_{3}$ with $p \in \mathbb{K}^{*}$.

Proof: It is sufficient to consider the basis change $\phi: \mathfrak{g} \rightarrow \mathfrak{g}$ defined by $e_{1}=\phi\left(w_{1}\right)=w_{1}$; $e_{2}=\phi\left(w_{2}\right)=\frac{1}{c_{1,2}^{1}} w_{2} ; \phi\left(w_{3}\right)=w_{3}$ and denote $p=\frac{c_{2,3}^{3}}{c_{1,2}^{1}}$.

Remark 2 From here on, $\mathfrak{g}_{2}(p)$ with $p \in \mathbb{K}^{*}$, will denote the 3-dimensional Lie algebra of law: $\left[e_{1}, e_{2}\right]=e_{1} ;\left[e_{2}, e_{3}\right]=p_{3}$, obtained in Proposition 2. 
Proposition 3 Let us consider the Lie algebra $\mathfrak{g}=\left\langle w_{1}, w_{2}, w_{3}\right\rangle:\left[w_{1}, w_{2}\right]=c_{1,2}^{1} w_{1}+$ $c_{1,2}^{2} w_{2} ;\left[w_{1}, w_{3}\right]=c_{1,3}^{3} w_{3} ;\left[w_{2}, w_{3}\right]=c_{2,3}^{3} w_{3}$, with $c_{1,2}^{1}, c_{1,2}^{2}, c_{1,3}^{3}, c_{2,3}^{3} \in \mathbb{K}^{*}$ associated with the configuration c) in Figure 1. Then, there exists a basis $\left\{e_{i}\right\}_{i=1}^{3}$ of $\mathfrak{g}$ with respect to which $\left[e_{1}, e_{2}\right]=p\left(e_{1}-e_{2}\right) ;\left[e_{1}, e_{3}\right]=e_{3} ;\left[e_{2}, e_{3}\right]=e_{3}$, with $p \in \mathbb{K}^{*}$.

Proof: It is sufficient to consider the basis change $\phi: \mathfrak{g} \rightarrow \mathfrak{g}$ defined by $e_{1}=\phi\left(w_{1}\right)=$ $\frac{1}{c_{1,3}^{3}} w_{1} ; e_{2}=\phi\left(w_{2}\right)=\frac{1}{c_{2,3}^{3}} w_{2} ; e_{3}=\phi\left(w_{3}\right)=w_{3}$, denote $p=\frac{c_{1,2}^{1}}{c_{2,3}^{3}}$ and keep in mind that $c_{1,2}^{1} c_{1,3}^{3}+c_{1,2}^{2} c_{2,3}^{3}=0$, due to the Jacobi identity.

Remark 3 From here on, $\mathfrak{g}_{3}(p)$ with $p \in \mathbb{K}^{*}$, will denote the 3-dimensional Lie algebra of law $\left[e_{1}, e_{2}\right]=p\left(e_{1}-e_{2}\right) ;\left[e_{1}, e_{3}\right]=e_{3} ;\left[e_{2}, e_{3}\right]=e_{3}$, obtained in Proposition 3.

Proposition 4 Let us consider the Lie algebra $\mathfrak{g}=\left\langle w_{1}, w_{2}, w_{3}\right\rangle:\left[w_{1}, w_{2}\right]=c_{1,2}^{1} w_{1}+$ $c_{1,2}^{2} w_{2} ;\left[w_{1}, w_{3}\right]=c_{1,3}^{1} w_{1}+c_{1,3}^{3} w_{3} ;\left[w_{2}, w_{3}\right]=c_{2,3}^{2} w_{2}+c_{2,3}^{3} w_{3}$, with $c_{1,2}^{1}, c_{1,2}^{2}, c_{1,3}^{1}, c_{1,3}^{3}, c_{2,3}^{2}$, $c_{2,3}^{3} \in \mathbb{K}^{*}$ associated with the configuration d) in Figure 1. Then

i) There exists a basis $\left\{e_{i}\right\}_{i=1}^{3}$ of $\mathfrak{g}$ verifying $\left[e_{1}, e_{2}\right]=-\frac{p_{1}}{p_{2}}\left(e_{1}-e_{2}\right) ;\left[e_{1}, e_{3}\right]=$ $p_{1} e_{1}+e_{3} ;\left[e_{2}, e_{3}\right]=p_{2} e_{2}+e_{3}$, with $p_{1}, p_{2} \in \mathbb{K}^{*}$.

ii) There exists a basis $\left\{v_{i}\right\}_{i=1}^{3}$ of $\mathfrak{g}$ verifying $\left[e_{1}, e_{2}\right]=-p\left(e_{1}-e_{2}\right) ;\left[e_{1}, e_{3}\right]=e_{1}+e_{3}$; $\left[e_{2}, e_{3}\right]=\frac{1}{p} e_{2}+e_{3}$, with $p \in \mathbb{K}^{*}$.

Proof: For i), it is sufficient to consider the basis change $\phi: \mathfrak{g} \rightarrow \mathfrak{g}$ given by $e_{1}=$ $\phi\left(w_{1}\right)=\frac{1}{c_{1,3}^{3}} w_{1} ; e_{2}=\phi\left(w_{2}\right)=\frac{1}{c_{2,3}^{3}} w_{2} ; e_{3}=\phi\left(w_{3}\right)=\frac{1}{c_{1,2}^{2}} w_{3}$ and the Jacobi identity $J\left(e_{1}, e_{2}, e_{3}\right)=0$, as well as denoting $p_{1}=\frac{c_{1,3}^{1}}{c_{1,2}^{2}}$ and $p_{2}=\frac{c_{2,3}^{2}}{c_{1,2}^{2}}$. Starting from this law and considering the basis change $\psi: \mathfrak{g} \rightarrow \mathfrak{g}$ with $e_{1}=\psi\left(w_{1}\right)=w_{1} ; e_{2}=\psi\left(w_{2}\right)=w_{2}$; $e_{3}=\psi\left(w_{3}\right)=\frac{1}{p_{1}} w_{3}$, we obtain the law stated in ii) after denoting $p=\frac{p_{1}}{p_{2}}$.

Remark 4 From here on, $\mathfrak{g}_{4}(p)$ with $p \in \mathbb{K}^{*}$, will denote the 3-dimensional Lie algebra of law $\left[e_{1}, e_{2}\right]=-p\left(e_{1}-e_{2}\right) ;\left[e_{1}, e_{3}\right]=e_{1}+e_{3} ;\left[e_{2}, e_{3}\right]=\frac{1}{p} e_{2}+e_{3}$, obtained in Proposition 4.

Proposition 5 The dimension of the derived Lie algebra $\mathcal{D}\left(\mathfrak{g}_{i}\right)=\left[\mathfrak{g}_{i}, \mathfrak{g}_{i}\right]$ is

$$
\operatorname{dim}\left(\mathcal{D}\left(\mathfrak{g}_{i}\right)\right)=\left\{\begin{array}{l}
1, \text { if } i=1 \\
2, \text { if } i=2,3 \vee(i=4 \wedge p=1) \\
3, \text { if } i=4 \text { with } p \neq 1
\end{array}\right.
$$

Proof: In virtue of Propositions 1, 2, 3 and 4 , we only need to study $\mathcal{D}\left(\mathfrak{g}_{4}\right)=\left\langle-p\left(e_{1}-\right.\right.$ $\left.\left.e_{2}\right), e_{1}+e_{3}, \frac{1}{p} e_{2}+e_{3}\right\rangle$. The coefficient matrix is

$$
\left(\begin{array}{ccc}
-p & 1 & 0 \\
p & 0 & \frac{1}{p} \\
0 & 1 & 1
\end{array}\right)
$$

whose rank equal to 2 if and only if $p=1$. 
Corollary 2 The following two statements are verified

1. $\mathfrak{g}_{1}$ is not isomorphic to $\mathfrak{g}_{2}(p), \mathfrak{g}_{3}(p)$ or $\mathfrak{g}_{4}(p)$, for $p \in \mathbb{K}^{*}$. Consequently, the last three are not isomorphic to Lie algebras associated with configurations having an isolated vertex.

2. Given $p \in \mathbb{K}^{*} \backslash\{1\}, \mathfrak{g}_{4}(p)$ is not isomorphic to $\mathfrak{g}_{2}(q)$, $\mathfrak{g}_{3}(q)$ either $\mathfrak{g}_{4}(1)$, for $q \in \mathbb{K}^{*}$.

Proposition 6 Given $p_{1}, p_{2} \in \mathbb{K}^{*}$ and $i \in\{2,3,4\}$, the Lie algebras $\mathfrak{g}_{i}\left(p_{1}\right)$ and $\mathfrak{g}_{i}\left(p_{2}\right)$ are isomorphic if and only if $p_{1}=p_{2}$ or $p_{1} \cdot p_{2}=1$.

Proof: Fixed and given $i \in\{2,3,4\}$, the Lie algebras $\mathfrak{g}_{i}\left(p_{1}\right)$ and $\mathfrak{g}_{i}\left(p_{2}\right)$ are isomorphic if and only if there exists a basis change leading from the law of $\mathfrak{g}_{i}\left(p_{1}\right)$ to the one of $\mathfrak{g}_{i}\left(p_{2}\right)$. Let $\left\{e_{j}\right\}_{j=1}^{3}$ and $\left\{w_{j}\right\}_{j=1}^{3}$ be the bases giving the law of $\mathfrak{g}_{i}\left(p_{1}\right)$ and $\mathfrak{g}_{i}\left(p_{2}\right)$, respectively and let us consider a general basis change given by $w_{1}=\sum a_{1, j} e_{j}, w_{2}=$ $\sum a_{2, j} e_{j}, w_{3}=\sum a_{3, j} e_{j}$. Imposing the law of $\mathfrak{g}_{i}\left(p_{1}\right)$ over $\mathfrak{g}_{i}\left(p_{2}\right)$, we obtain a system whose solutions are $p_{1}=p_{2}$ or $p_{1} \cdot p_{2}=1$.

Proposition 7 Given $p \in \mathbb{K}^{*}$, Lie algebra $\mathfrak{g}_{2}(p)$ is isomorphic to Lie algebra $\mathfrak{g}_{3}(p)$.

Proof: The isomorphism is $\phi: \mathfrak{g}_{2}(p) \rightarrow \mathfrak{g}_{3}(p)$ defined by $w_{1}=\phi\left(e_{1}\right)=-e_{2}+e_{3}$; $w_{2}=\phi\left(e_{2}\right)=-e_{2}-e_{3} ; w_{3}=\phi\left(e_{3}\right)=e_{1}$; where $\left\{e_{i}\right\}_{i=1}^{3}$ and $\left\{w_{i}\right\}_{i=1}^{3}$ are the respective bases of $\mathfrak{g}_{2}(p)$ and $\mathfrak{g}_{3}(p)$.

Remark 5 Proposition 7 involves that configurations of Figure 3 are associated with the same Lie algebra for a given $p$.
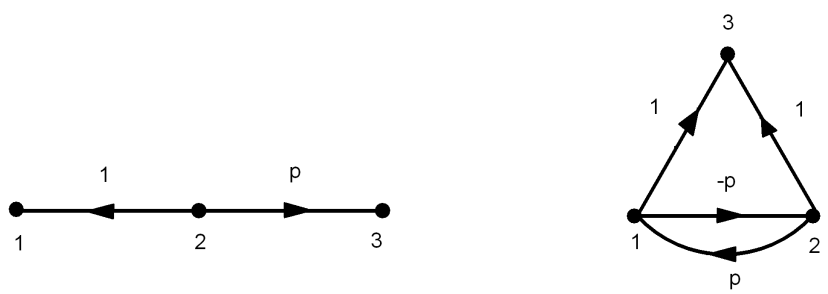

Figure 3: Isomorphism from Proposition 7.

Proposition 8 Lie algebra $\mathfrak{g}_{2}(-1)$ is isomorphic to Lie algebra $\mathfrak{g}_{4}(1)$.

Proof: The isomorphism is given by $\phi: \mathfrak{g}_{2}(-1) \rightarrow \mathfrak{g}_{4}(1)$, where $w_{1}=\phi\left(e_{1}\right)=e_{1}-$ $e_{2} ; w_{2}=\phi\left(e_{2}\right)=-e_{2}+e_{3} ; w_{3}=\phi\left(e_{3}\right)=e_{1}+e_{2}+e_{3}$. 

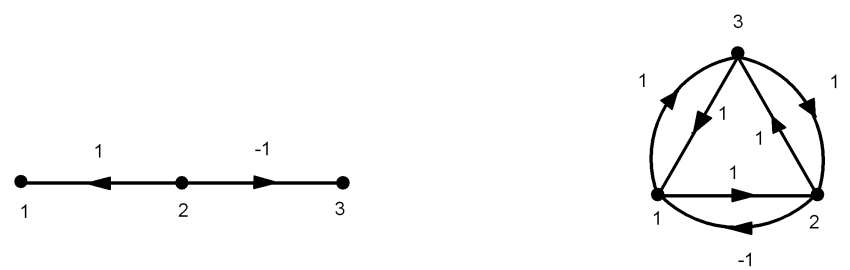

Figure 4: Isomorphism from Proposition 8.

Remark 6 Proposition 8 establishes that configurations in Figure 4 correspond to the same Lie algebra.

Proposition $9 \mathfrak{s l}_{2}(\mathbb{K})$ is isomorphic to $\mathfrak{g}_{4}(p)$ if and only if $p=-1$.

Proof: Given $p \in \mathbb{K}^{*} \backslash\{1\}$, we consider the Lie algebra $\mathfrak{g}_{4}(p)=\left\langle e_{1}, e_{2}, e_{3}\right\rangle$ with law $\left[e_{1}, e_{2}\right]=-p\left(e_{1}-e_{2}\right) ;\left[e_{1}, e_{3}\right]=e_{1}+e_{3} ;\left[e_{2}, e_{3}\right]=\frac{1}{p} e_{2}+e_{3}$ and Lie algebra $\mathfrak{s l}_{2}(\mathbb{K})=\left\langle w_{1}, w_{2}, w_{3}\right\rangle$ with law $\left[w_{1}, w_{2}\right]=2 w_{2} ;\left[w_{1}, w_{3}\right]=-2 w_{3} ;\left[w_{2}, w_{3}\right]=w_{1}$. When defining an arbitrary basis change $e_{i}=\sum_{j=1}^{3} a_{i, j} w_{j}$, for $i=1,2,3$ and imposing the laws of $\mathfrak{g}_{4}(p)$ and $\mathfrak{s l}_{2}(\mathbb{K})$, we obtain a system of equations such that every solution involves $p=-1$, which concludes the proof.

Remark 7 Proposition 9 implies that configurations in Figure 5 comes from the same Lie algebra.
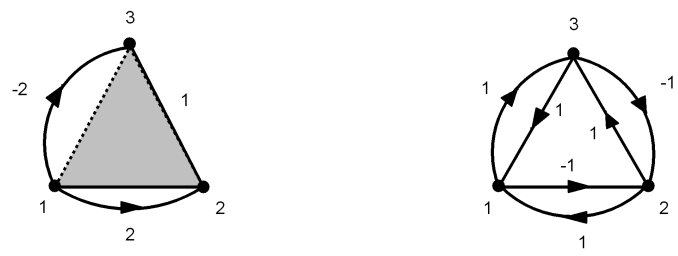

Figure 5: Isomorphism from Proposition 9.

All the previous results can be summarized as follows

Theorem 1 The isomorphism classes of 3-dimensional Lie algebras are the following
a) $\mathfrak{g}_{1}$.
b) $\mathfrak{g}_{2}(-1) \cong \mathfrak{g}_{3}(-1) \cong \mathfrak{g}_{4}(1)$. 
c) $\mathfrak{g}_{2}(p) \cong \mathfrak{g}_{2}\left(\frac{1}{p}\right) \cong \mathfrak{g}_{3}(p) \cong \mathfrak{g}_{3}\left(\frac{1}{p}\right), \forall p \in \mathbb{K}^{*} \backslash\{-1\}$.

d) $\mathfrak{g}_{4}(-1) \cong \mathfrak{s l}_{2}(\mathbb{K})$.

e) $\mathfrak{g}_{4}(p) \cong \mathfrak{g}_{4}\left(\frac{1}{p}\right), \forall p \in \mathbb{K}^{*} \backslash\{-1,1\}$.

Moreover, the algebras belonging to the first three classes are 2-step solvable and non-nilpotent, while the corresponding with the fourth class is simple.

\section{Algorithmic methods}

In this section we show two algorithms dealing with converse questions: the first is devoted to obtain the digraph associated with a given Lie algebra starting from its law; and the second is useful to determine if a weighted digraph is associated with a Lie algebra or not.

\subsection{Algorithm to obtain the digraph associated with a Lie algebra}

Given an $n$-dimensional Lie algebra $\mathfrak{g}$ with basis $\mathcal{B}_{n}=\left\{e_{i}\right\}_{i=1}^{n}$, its law consists only of brackets $\left[e_{i}, e_{j}\right]=c_{i, j}^{i} e_{i}+c_{i, j}^{j} e_{j}$. This is because of dealing with digraphs and not with full triangles.

To implement the algorithm, we have used the symbolic computation package MAPLE 12, loading the libraries linalg, GraphTheory and Maplets [Elements]. The first two libraries allow us to apply commands of Linear Algebra and Graph Theory, respectively; whereas the last is used to display a message so that the user introduces the required input in the first subroutine, devoted to define the law of the Lie algebra $\mathfrak{g}$. The algorithm to obtain the digraph associated with $\mathfrak{g}$ considers the following two steps:

1. Entering the law of $\mathfrak{g}$ by means of a routine computing the Lie bracket between two arbitrary basis vectors in $\mathcal{B}_{n}$.

2. Defining the digraph associated with $\mathfrak{g}$ using the method reviewed in Section 3 .

The first routine, named law, receives two natural numbers as inputs. These numbers represent the subindexes of two basis vectors in $\mathcal{B}_{n}$. The subroutine returns the result of the bracket between these two vectors. In addition, conditional sentences are inserted to determine the non-zero brackets and the skew-symmetry property. Since the user has to complete the subroutine inserting the non-zero brackets of $\mathfrak{g}$, we have also added a sentence at the beginning of the implementation, reminding this fact. Note that before running any other sentence, we must restart all the variables and delete all the computations saved for previous law. Additionally, we must update the value of variable dim with the dimension of $\mathfrak{g}$. 


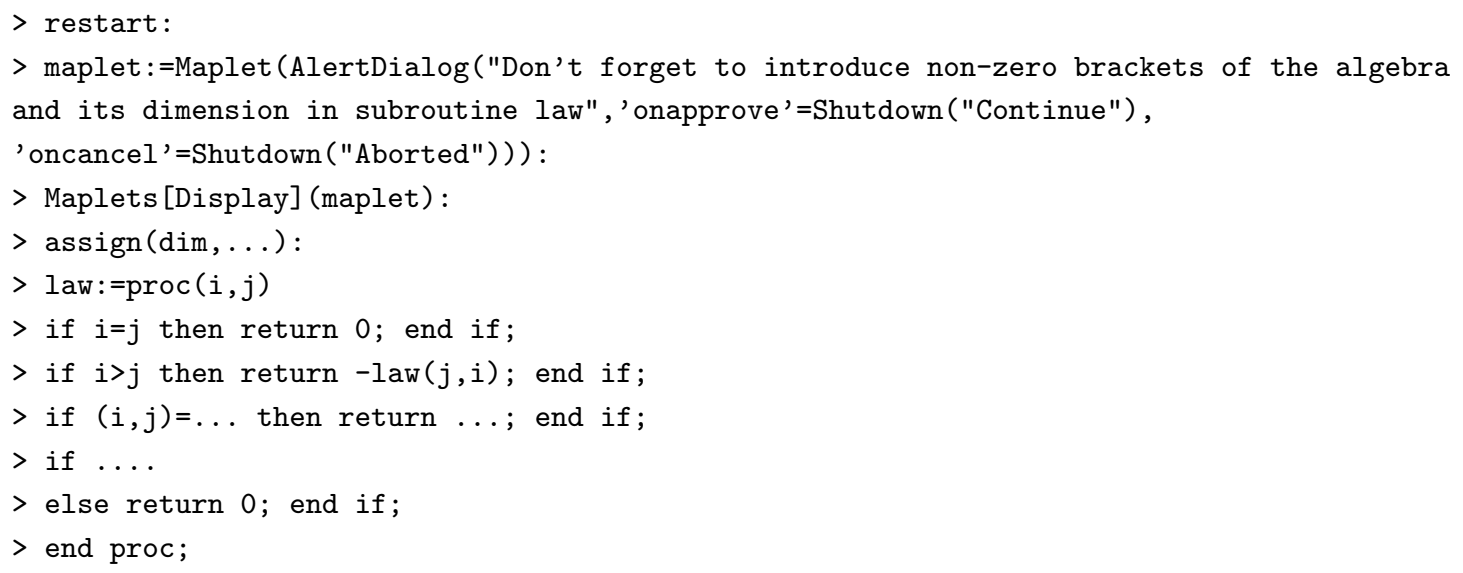

The ellipsis in command assign corresponds to write the dimension of $\mathfrak{g}$. The following two suspension points are associated with the computation of $\left[e_{i}, e_{j}\right]$ : first, the value of the subindexes $(i, j)$ and second, the result of $\left[e_{i}, e_{j}\right]$ with respect to $\mathcal{B}_{n}$. The last ellipsis denotes the rest of non-zero brackets. For each non-zero bracket, a new sentence if has to be included in the cluster.

Next, we implement the second step of the algorithm with the routine drawdigraph, receiving the dimension $\mathrm{n}$ of $\mathfrak{g}$ as input. This routine draws the digraph associated with $\mathfrak{g}$. To do so, two local variables $\mathrm{V}$ and $\mathrm{E}$ have been defined: $\mathrm{V}$ is a list with the vertices of the digraph and $\mathrm{E}$ is a set containing the edges. Hence, several loops are programmed to include all the directed, weighted edges in the set $\mathrm{E}$ according to the non-zero brackets saved in the subroutine law.

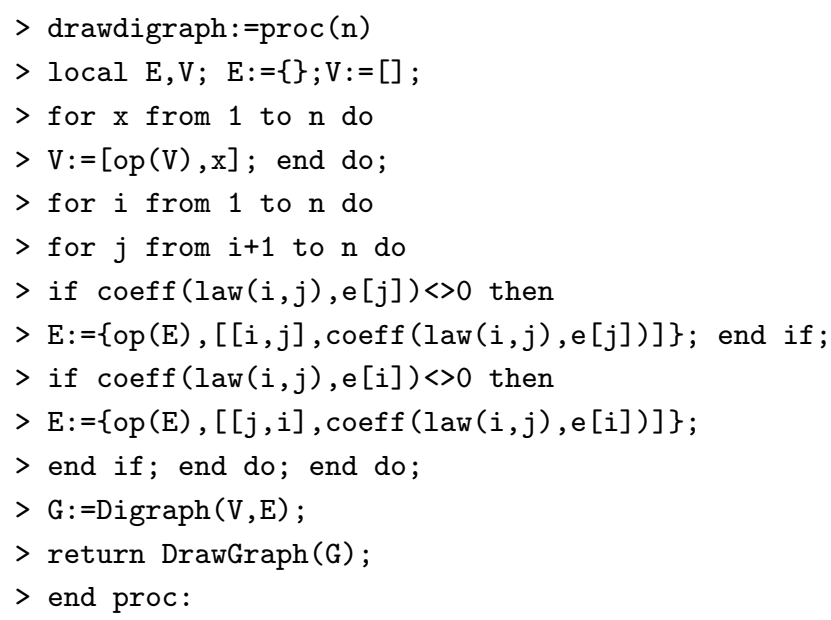

Example 1 To illustrate this algorithm, we apply it to the 6-dimensional Lie algebra with law $\left[e_{1}, e_{3}\right]=2 e_{3},\left[e_{1}, e_{4}\right]=-e_{4},\left[e_{1}, e_{6}\right]=e_{6},\left[e_{2}, e_{3}\right]=-e_{3},\left[e_{2}, e_{4}\right]=e_{4}$, $\left[e_{2}, e_{5}\right]=e_{5}$. First, we complete the routine law as follows:

$>$ restart: 


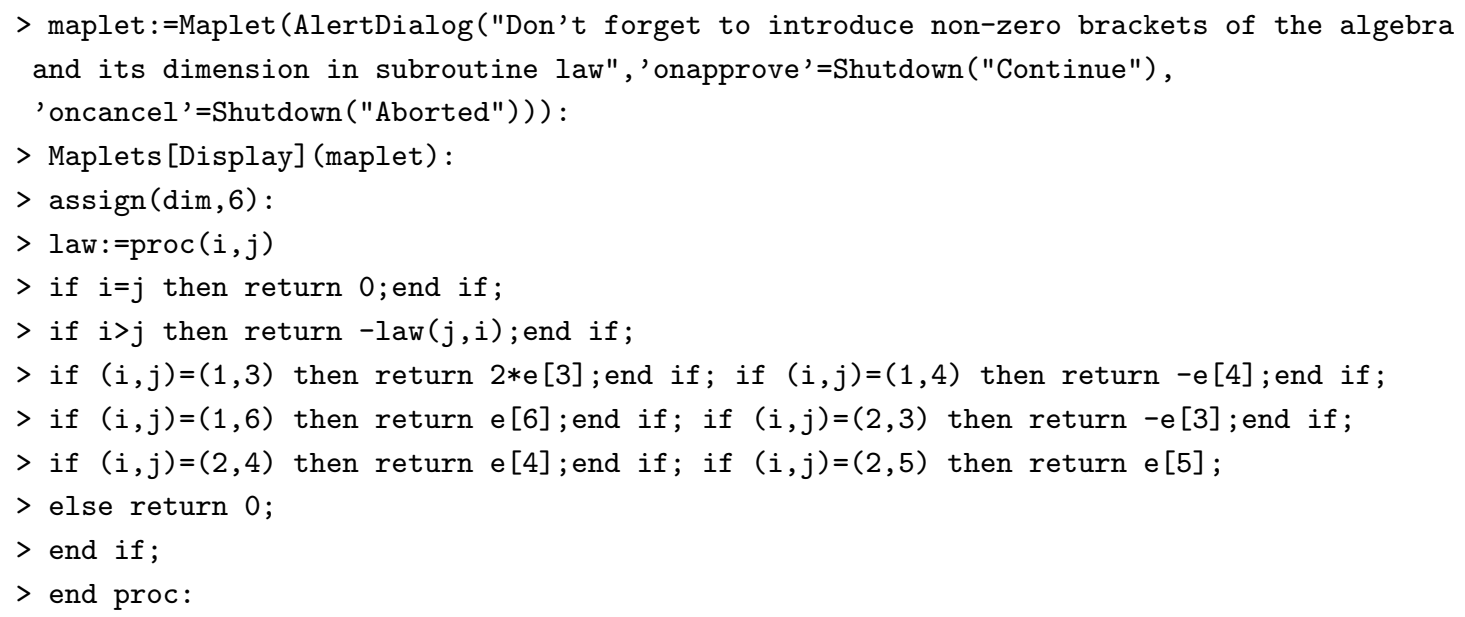

Next, we run the routine drawdigraph and execute the sentence drawdigraph (dim) obtaining the digraph in Figure 6.

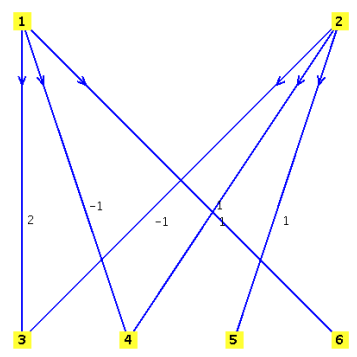

Figure 6: output from Example 1.

\subsection{Algorithm to decide if a digraph is associated with a Lie algebra}

We show an algorithmic procedure to determine if a given digraph is associated or not with a Lie algebra. The algorithm consists of two steps: a) generating the law candidate to be a Lie algebra using the construction reviewed in Section 3; and b) checking if the Jacobi identities are satisfied for this law. To implement the algorithm, we need load the libraries DifferentialGeometry, LieAlgebras and GraphTheory to activate commands related to Lie algebras and Graph Theory.

First, we build a vector space associated with the digraph using the routine program, receiving two inputs: a list $\mathrm{V}$ with the vertices of the digraph and a set $\mathrm{E}$ with the directed, weighted edges. As output, we obtain a vector space with basis $\left\{e_{i}\right\}_{i=1}^{n}$ where $e_{i}$ corresponds to vertex $i$ from the list $\mathrm{V}$ and the brackets associated with the edges in the set $\mathrm{E}$. To implement this routine we define two local variables: $\mathrm{B}$ and $\mathrm{L}$, where $\mathrm{B}$ saves the basis $\left\{e_{i}\right\}_{i=1}^{n}$ and $\mathrm{L}$ is a list containing the indexes of the structure constants from the non-zero brackets. 
J. Cáceres, M. Ceballos, J. Núñez, M.L. Puertas, Á.F.Tenorio

$>\operatorname{program}:=\operatorname{proc}(\mathrm{V}, \mathrm{E})$

$>$ local $\mathrm{B}, \mathrm{L}$;

$>\mathrm{B}:=[] ; \mathrm{L}:=[]$;

$>$ for $x$ from 1 to nops(V) do

$>\mathrm{B}:=[\mathrm{op}(\mathrm{B}), \mathrm{e}[\mathrm{x}]]$;

$>$ end do;

$>$ for $i$ from 1 to nops(E) do

$>\mathrm{L}:=[\mathrm{op}(\mathrm{L}),[[\mathrm{op}(\mathrm{E}[\mathrm{i}][1]), \mathrm{E}[\mathrm{i}][1][2]], \mathrm{E}[\mathrm{i}][2]]]$;

$>$ end do;

$>$ return_DG([["LieAlgebra",Alg1, $[\operatorname{nops}(\mathrm{V})]], \mathrm{L}])$;

$>$ end proc:

Next, the vector space having such basis and law is generated when evaluating the sentence

$>\operatorname{DGsetup}(\operatorname{program}(\mathrm{V}, \mathrm{E}))$;

After defining this vector space Alg1, we can operate over it. More concretely, we check if Jacobi identities hold or not for Alg1:

Alg1 > Query (Alg1, "Jacobi");

The vector space Alg1 defined by the output of program is a Lie algebra if and only if the answer is true for this question.

Example 2 Consider the digraph in Figure \%. After running the routine program, we define the list $\mathrm{V}$ of vertices and the set $\mathrm{E}$ of edges. Then the routine program generates the vector space associated with the graph and finally Jacobi identities are checked

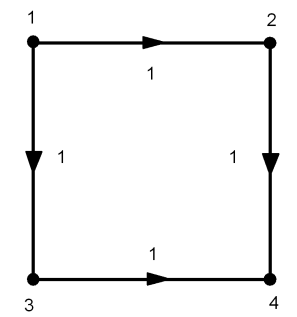

Figure 7: Digraph of Example 2.

$>\mathrm{V}:=[1,2,3,4]$;

$>\mathrm{E}:=\{[[1,2], 1],[[1,3], 1],[[2,4], 1],[[3,4], 1]\}$;

$>\operatorname{DGsetup}(\operatorname{program}(\mathrm{V}, \mathrm{E}))$;

Alg1 > Query(Alg1, "Jacobi");

$>\quad$ false

Since the answer is false, the digraph in Figure 7 is not associated with a 4dimensional Lie algebra. 


\section{Acknowledgements}

This work has been partially supported by MTM2010-19336 and FEDER.

\section{References}

[1] A. Carriazo, L.M. Fernández and J. NúÑez, Combinatorial structures associated with Lie algebras of finite dimension, Linear Algebra and Applications. 389 (2004), 43-61.

[2] M. Ceballos, J. Núñez And A.F. Tenorio, Complete triangular structures and Lie algebras, International Journal of Computer Mathematics (2011), 1-13. DOI: 10.1080/00207161003767994.

[3] M. Ceballos, J. Núñez and A.F. Tenorio, Study of Lie algebras by using combinatorial structures, Linear Algebra and its Applications (2011), DOI: 10.1016/j.laa.2010.11.030.

[4] V.S. VARAdarajan, Lie Groups, Lie Algebras and Their Representations, Springer, 1984.

[5] H.S. Wilf, Algorithms and Complexity, Prentice Hall, 1986. 